\title{
Extended interacting boson model description of Pd nuclei in the A $\sim 100$ transitional region
}

\author{
M. Böyükata'1,2,a , E. Ellinger ${ }^{1}$, C. Fransen ${ }^{1}$, and J. Jolie ${ }^{1}$ \\ ${ }^{1}$ Institut für Kernphysik, Universität zu Köln, D-50937 Köln, Germany \\ ${ }^{2}$ Department of Elementary Science Education, Faculty of Education, Canakkele Onsekiz Mart University, \\ TR-17100, Çanakkele, Turkey
}

\begin{abstract}
.
Studies of even-even nuclei in the A 100 transitional mass region within the framework of the interacting boson model-1 (IBM-1) have been expanded down to ${ }^{98} \mathrm{Pd}$ nuclei to compare the calculation with new experimental results from measurements obtained at the Institute of Nuclear Physics in Cologne. The low-lying energy levels and the E2 transition rates of ${ }^{98-100} \mathrm{Pd}$ nuclei are investigated and their geometric structures are described in the present work. We have also focused on the new $\mathrm{B}\left(\mathrm{E} 2: 2_{1}^{+} \rightarrow 0_{1}^{+}\right)$values of ${ }^{112,114} \mathrm{Pd}$ nuclei to compare with previously calculated values.
\end{abstract}

\section{Introduction}

The structure of the even-even nuclei in the A 100 region were studied both theoretically and experimentally during the last years [1-9]. The ratio of the energy of the $4^{+}$over $2^{+}$in the ground state band, $E_{4^{+}} / E_{2^{+}}$, is always used to investigste whether the nuclei are spherical or not. Three possible dynamical symmetries, $\mathrm{U}(5), \mathrm{SU}(3), \mathrm{O}(6)$, of the interacting boson model (IBM) [10] are associated with these ratios; $E_{4^{+}} / E_{2^{+}}=2.0,3.33,2.5$ indicate spherical, axially deformed, $\gamma$-unstable nuclei, respectively. It is also possible to get hints for the geometry of nuclei by plotting potential energy surface in terms of deformation parameters $\beta$ and $\gamma$. Actually, the even-even $\mathrm{Pd}, \mathrm{Ru}$ and Mo nuclei around the $\mathrm{N}=50$ shell show the spherical behaviour according to the interacting boson model-1 (IBM-1) calculation in Ref. [6].

The main purpose of this paper is to complete the investigations of all nuclei in the isotopic chain of $\mathrm{Pd}$ with $\mathrm{Z}=46$ by expanding the previous work [6]. The energy levels and $\mathrm{B}(\mathrm{E} 2)$ values of ${ }^{98,100} \mathrm{Pd}$ isotopes have been calculated with refitted IBM-1 parameters and compared to new experimental results $[8,9]$. The new experimental results for the $\mathrm{B}\left(\mathrm{E} 2: 2_{1}^{+} \rightarrow 0_{1}^{+}\right)$values of ${ }^{112,114} \mathrm{Pd}$ nuclei $[1,2]$ are compared to the earlier experimental data and the calculated ones, too. We have also briefly mentioned about the new experiment on ${ }^{98} \mathrm{Pd}$ nucleus in this work but the detailed discussion will be presented in a forthcoming paper [14]

\footnotetext{
a e-mail: boyukata@comu.edu.tr
} 


\section{The Interacting Boson Model}

The interacting boson model [10], based on the group theoretical approaches, is useful to describe the properties of collective phenomena in the valance space. This semi-microscopic theory describes even-even nuclei by $N$ bosons with $N$ equal to the number of valence nucleons divided by two. In the original version of IBM-1 (no distinction between neutron and proton bosons), applicable to eveneven nuclei, the basic building blocks are $s$ and d bosons, with angular momentum $L=0$ and $L=2$. It is successful in the description of energy spectra and also electromagnetic transition strengths in a wide range of the nuclear chart. In the present calculation, the following multipole form of the Hamiltonian in the sd-boson space has been used

$$
\hat{H}=\epsilon \hat{n}_{d}+\kappa \hat{Q} \cdot \hat{Q}+\kappa^{\prime} \hat{L} \cdot \hat{L}+\hat{q}_{3} \hat{T}_{3} \cdot \hat{T}_{3}+\hat{q}_{4} \hat{T}_{4} \cdot \hat{T}_{4},
$$

where $\hat{n}_{d}, \hat{Q}, \hat{L}, \hat{T}_{3}$ and $\hat{T}_{4}$ terms are the d-boson number operator, the quadrupole operator, the angular momentum operator and the octupole and hexadecapole operators, respectively, and they are defined in terms of $\mathrm{s}$ and $\mathrm{d}$ bosons [10]

$$
\begin{aligned}
\hat{n}_{d} & =\sqrt{5}\left[d^{\dagger} \times \tilde{d}\right]_{0}^{(0)}, \quad \hat{L}=\sqrt{10}\left[d^{\dagger} \times \tilde{d}\right]^{(1)}, \\
\hat{Q} & =\left[d^{\dagger} \times \tilde{s}+s^{\dagger} \times \tilde{d}\right]^{(2)}+\chi\left[d^{\dagger} \times \tilde{d}\right]^{(2)}, \\
\hat{T}_{3} & =\left[d^{\dagger} \times \tilde{d}\right]^{(3)} \quad \hat{T}_{4}=\left[d^{\dagger} \times \tilde{d}\right]^{(4)} .
\end{aligned}
$$

The electric quadrupole transition rates in the IBM-1 have been calculated with $\hat{T}(\mathrm{E} 2)=e_{\mathrm{b}} \cdot \hat{Q}$, and by using the expression for the quadrupole operator $\hat{Q}$ from (2) we deduce as

$$
\hat{T}(\mathrm{E} 2)=e_{\mathrm{b}}\left[\left(d^{\dagger} \times \tilde{s}+s^{\dagger} \times \tilde{d}\right)^{(2)}+\chi\left(d^{\dagger} \times \tilde{d}\right)\right]^{(2)}
$$

where $e_{\mathrm{b}}$ is the boson effective charge and $\chi$ is another free parameter.

A geometric shape visualization of the even-even nuclei is made by plotting the potential energy surface in the $(\beta, \gamma)$ plane. The $\mathrm{V}(\beta, \gamma)$ can be obtained by calculating the expectation value of the Hamiltonian (1) in the coherent state [13] and is given in the following form

$$
\begin{aligned}
V(\beta, \gamma)= & \epsilon \frac{N \beta^{2}}{1+\beta^{2}}+\kappa N\left[\frac{5+\left(1+\chi^{2}\right) \beta^{2}}{1+\beta^{2}}+\frac{(N-1)\left(\frac{2 \chi^{2} \beta^{4}}{7}+4 \sqrt{\frac{2}{7}} \chi \beta^{3} \cos (3 \gamma)+4 \beta^{2}\right)}{\left(1+\beta^{2}\right)^{2}}\right] \\
& +\kappa^{\prime} \frac{6 N \beta^{2}}{1+\beta^{2}}+q_{3} \frac{7 N\left(5+\chi^{2}\right)}{5} \frac{\beta^{2}}{1+\beta^{2}}+q_{4}\left[\frac{9 N}{5} \frac{\beta^{2}}{1+\beta^{2}}+\frac{18 N(N-1)}{35} \frac{\beta^{4}}{\left(1+\beta^{2}\right)^{2}}\right]
\end{aligned}
$$

where $N$ is number of bosons, $\beta, \gamma$ are deformation parameters (usually, $\beta \geq 0,0^{\circ} \leq \gamma \leq 60^{\circ}$ ) and other terms are the same as in the Hamiltonian (1). For the $\beta=0$, nucleus is spherical, for the $\beta>0$ it is axially deformed and in this case for $\gamma=0^{\circ}, 30^{\circ}, 60^{\circ}$ the nucleus has correspondingly prolate, triaxial, oblate shape.

\section{New experimental data on ${ }^{98} \mathrm{Pd}$}

An experiment on ${ }^{98} \mathrm{Pd}$ using the recoil distance Doppler-shift method (RDDS) has been performed at the Cologne TANDEM accelerator with the Cologne plunger device [9]. The aim of the experiment was to deduce for the first time $E 2$ transition strengths between the lowest yrast states in ${ }^{98} \mathrm{Pd}$ from measured lifetimes of these states. The fusion-evaporation reaction ${ }^{92} \mathrm{Mo}\left({ }^{10} \mathrm{~B}, 3 \mathrm{np}\right){ }^{98} \mathrm{Pd}$ was used to produce the excited ${ }^{98} \mathrm{Pd}$ nuclei with a beam energy of $E\left({ }^{10} \mathrm{~B}\right)=54 \mathrm{MeV}$. To determine the lifetimes 

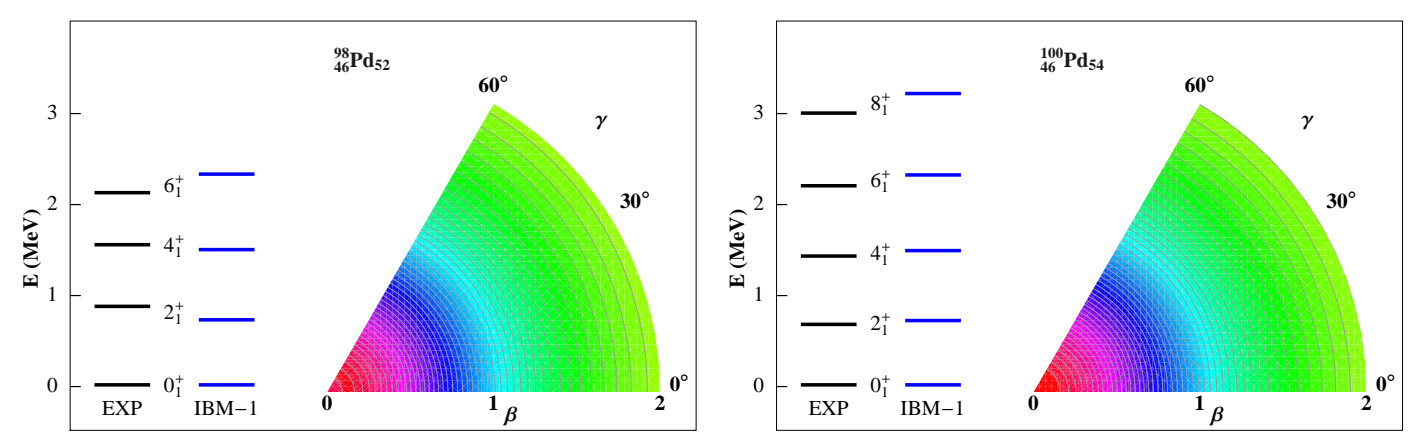

Figure 1. The experimental $[8,9,14]$ and calculated energy spectrum. The potential energy surface in $\beta, \gamma$ plane of ${ }^{98-100} \mathrm{Pd}$ nuclei are also given in the right side of each panel.

Table 1. Hamiltonian parameters used in the ibm1-code [12] (in units of $\mathrm{keV}$ and $\chi=0.02$ ).

\begin{tabular}{llllll}
\hline & $\epsilon$ & $\kappa$ & $\kappa^{\prime}$ & $q_{3}$ & $q_{4}$ \\
\hline${ }^{98} \mathrm{Pd}$ & 580 & 4.0 & 8.7 & 14.6 & 34.1 \\
${ }^{100} \mathrm{Pd}$ & 636 & -1.0 & 7.1 & 5.3 & 12.4 \\
\hline
\end{tabular}

the established Differential Decay Curve method (DDCM) was employed [11]. From the data of this experiment it was possible to determine five new E2 transition strengths in ${ }^{98} \mathrm{Pd}$ between yrast states with spin quantum numbers $\leq 10$.

\section{Discussion}

The best fitted parameters are given in Table 1 for the Hamiltonian (1). For the parametrization, a linear dependence on the fractional fillings is assumed by adding extra two nuclei to the calculation described in the previous work [6]. The energy levels of ${ }^{98-100} \mathrm{Pd}$ are calculated and the comparison of experimental spectra for the ground state illustrated in Fig. 1. Right sides of each panel also include the energy surfaces in $\beta, \gamma$ plane. It is clearly seen that the geometric shape of both nuclei are spherical since minimums of energies are found at $\beta \simeq 0$. Therefore these nuclei are close to the $\mathrm{U}(5)$ dynamical symmetry. In addition to the energy levels, the electric quadrupole transition rates in the IBM-1 have been calculated using the quadrupole transition operator $\hat{T}(E 2)$ given in Eq. (3). The boson effective charge $e_{\mathrm{b}}=0.097$ and $\chi=0.2$ are used for the calculation of $\mathrm{B}(\mathrm{E} 2)$ values of ${ }^{98-100} \mathrm{Pd}$ nuclei. These parameters are obtained from Ref. [6] and keeping the same values for overall calculations of all nuclei in the $A \sim 100$ region. The calculated B(E2) values are compared with experimental data and shown in Fig. 2. It is demonstrated in this figure that the new results (indicted with full blue symbols) for the $\mathrm{B}\left(\mathrm{E} 2: 2_{1}^{+} \rightarrow 0_{1}^{+}\right)$values of ${ }^{112,114} \mathrm{Pd}$ nuclei are more closer to the calculated ones than the earlier experimental data (indicted with open blue symbols). 


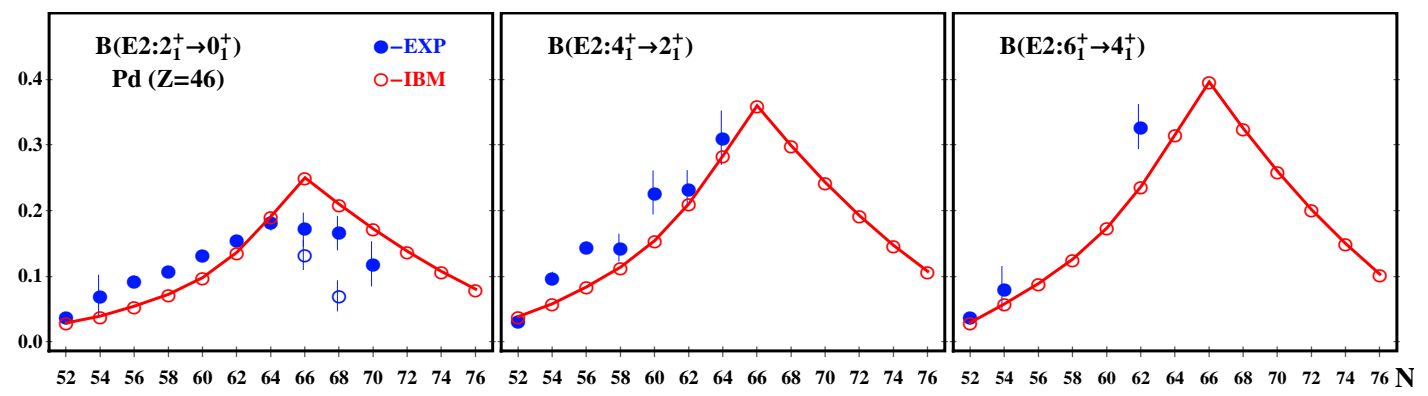

Figure 2. The experimental (blue) and calculated (red) $\mathrm{B}(\mathrm{E} 2)$ values in the Pd isotopes. The $\mathrm{B}\left(\mathrm{E} 2: 2_{1}^{+} \rightarrow 0_{1}^{+}\right)$, $\mathrm{B}\left(\mathrm{E} 2: 4_{1}^{+} \rightarrow 2_{1}^{+}\right), \mathrm{B}\left(\mathrm{E} 2: 6_{1}^{+} \rightarrow 4_{1}^{+}\right)$values of ${ }^{98-100} \mathrm{Pd}$ and the $\mathrm{B}\left(\mathrm{E} 2: 2_{1}^{+} \rightarrow 0_{1}^{+}\right)$values of ${ }^{112,114} \mathrm{Pd}$ are added to figures taken from Ref. [6]. The experimental data in Ref. [6] were taken from the National Nuclear Data Center [15].

In this paper, we have focused on the expansion of the IBM-1 calculation for Pd nuclei down to $N=52$. The low-lying energy levels ${ }^{98,100} \mathrm{Pd}$ nuclei are calculated with Hamiltonian parameters and and their geometric shapes are predicted by using the same Hamiltonian parameters in $V(\beta, \gamma)$ given by Eq. (4). The $\mathrm{B}(\mathrm{E} 2)$ values for the $2_{1}^{+} \rightarrow 0_{1}^{+}, 4_{1}^{+} \rightarrow 2_{1}^{+}$and $6_{1}^{+} \rightarrow 4_{1}^{+}$transitions of ${ }^{98,100} \mathrm{Pd}$ nuclei are calculated and compared with recent experimental data for ${ }^{98} \mathrm{Pd}[9,14]$ and ${ }^{100} \mathrm{Pd}[5,8]$. The $\mathrm{B}(\mathrm{E} 2)$ values of all nuclei in the $\mathrm{Z}=46$ isotopic chain with neutron numbers $52 \leq N \leq 76$ are reinvestigated to emphasize the behaviour of electromagnetic transition rates along the chain by adding more recent experimental $\mathrm{B}\left(\mathrm{E} 2: 2_{1}^{+} \rightarrow 0_{1}^{+}\right)$values of ${ }^{112,114} \mathrm{Pd}$ nuclei $[1,2]$.

\section{Acknowledgments}

This work is supported by the Scientific and Technical Research Council (TÜBİTAK) BIDDEB-2219.

\section{References}

[1] H. Machet, et al., JYFL Annual Report (2003).

[2] A. Dewald, et al., Phys. Rev. C 78, 051302 (2008).

[3] C. Fransen, et al., AIP Conf. Proc. 1090, 529-533 (2009).

[4] D. Radeck, et al., Nucl. Phys. A 8211 (2009)

[5] D. Radeck, et al., Phys. Rev. C 80, 044331 (2009)

[6] M. Böyükata, P. Van Isacker, and İ. Uluer, J. Phys. G: Nucl. Part. Phys. 37, 105102 (2010). AIP Conf. Proc. 1072, 223-228 (2008).

[7] M. Böyükata, İ. Uluer, AIP Conf. Proc. 1231, 201-202 (2009).

[8] V. Anagnostatou, et al., Appl. Rad. and Iso. 70, 1321 (2012).

[9] E. Ellinger, Diploma Thesis, University of Cologne (2012).

[10] F. Iachello and A. Arima, The Interacting Boson Model, Cambridge University Press, (1987).

[11] A. Dewald, S. Harissopulos, P. von Brentano, Z. Phys. A 334, 163 (1989).

[12] P. Van Isacker, ibm1-code (unpublished).

[13] J. N. Ginocchio and M. W. Kirson Phys. Rev. Lett. 44, 1744 (1980), A. E. L. Dieperink, O. Scholten and F. Iachello Phys. Rev. Lett.44, 1747 (1980)

[14] E. Ellinger, M. Böyükata, C. Fransen, J. Jolie, et al. in progress (2013).

[15] National Nuclear Data Center (NNDC) http://www.nndc.bnl.gov/ 